Louisiana State University

LSU Digital Commons

Faculty Publications

Department of Biological Sciences

$5-1-2016$

\title{
Natural isoforms of the Photosystem II D1 subunit differ in photoassembly efficiency of the water-oxidizing complex
}

\author{
David J. Vinyard \\ Waksman Institute of Microbiology \\ Jennifer S. Sun \\ Waksman Institute of Microbiology \\ Javier Gimpel \\ Division of Biological Sciences \\ Gennady M. Ananyev \\ Waksman Institute of Microbiology \\ Stephen P. Mayfield \\ Division of Biological Sciences
}

See next page for additional authors

Follow this and additional works at: https://digitalcommons.Isu.edu/biosci_pubs

\section{Recommended Citation}

Vinyard, D., Sun, J., Gimpel, J., Ananyev, G., Mayfield, S., \& Charles Dismukes, G. (2016). Natural isoforms of the Photosystem II D1 subunit differ in photoassembly efficiency of the water-oxidizing complex. Photosynthesis Research, 128 (2), 141-150. https://doi.org/10.1007/s11120-015-0208-8

This Article is brought to you for free and open access by the Department of Biological Sciences at LSU Digital Commons. It has been accepted for inclusion in Faculty Publications by an authorized administrator of LSU Digital Commons. For more information, please contact ir@lsu.edu. 


\section{Authors}

David J. Vinyard, Jennifer S. Sun, Javier Gimpel, Gennady M. Ananyev, Stephen P. Mayfield, and G. Charles Dismukes 


\title{
Natural isoforms of the Photosystem II D1 subunit differ in photoassembly efficiency of the water-oxidizing complex
}

\author{
David J. Vinyard ${ }^{1,2,4} \cdot$ Jennifer S. Sun ${ }^{1,5} \cdot$ Javier Gimpel $^{3,6} \cdot$ Gennady M. Ananyev $^{1,2}$ • \\ Stephen P. Mayfield ${ }^{3} \cdot$ G. Charles Dismukes ${ }^{1,2}$ (1)
}

Received: 16 September 2015/ Accepted: 23 November 2015/Published online: 19 December 2015

(C) Springer Science+Business Media Dordrecht 2015

\begin{abstract}
Oxygenic photosynthesis efficiency at increasing solar flux is limited by light-induced damage (photoinhibition) of Photosystem II (PSII), primarily targeting the D1 reaction center subunit. Some cyanobacteria contain two natural isoforms of D1 that function better under low light (D1:1) or high light (D1:2). Herein, rates and yields of photoassembly of the $\mathrm{Mn}_{4} \mathrm{CaO}_{5}$ water-oxidizing complex (WOC) from the free inorganic cofactors $\left(\mathrm{Mn}^{2+}, \mathrm{Ca}^{2+}\right.$, water, electron acceptor) and apo-WOC-PSII are shown to differ significantly: D1:1 apo-WOC-PSII exhibits a 2.3-fold faster ratelimiting step of photoassembly and up to seven-fold faster rate to the first light-stable $\mathrm{Mn}^{3+}$ intermediate, $\mathrm{IM}_{1}{ }^{*}$, but with a much higher rate of photoinhibition than D1:2. Conversely, D1:2 apo-WOC-PSII assembles slower but has up to sevenfold higher yield, achieved by a higher quantum yield of charge separation and slower photoinhibition rate. These
\end{abstract}

G. Charles Dismukes

dismukes@rci.rutgers.edu

1 Waksman Institute of Microbiology, Rutgers, The State University of New Jersey, 190 Frelinghuysen Rd.,

Piscataway, NJ 08854, USA

2 Department of Chemistry and Chemical Biology, Rutgers, The State University of New Jersey, Piscataway, NJ 08854, USA

3 San Diego Center for Algae Biotechnology, Division of Biological Sciences, University of California, San Diego, La Jolla, CA 92093, USA

4 Present Address: Department of Chemistry, Yale University, New Haven, CT 06520, USA

5 Present Address: Department of Molecular, Cellular, and Development Biology, Yale University, New Haven, CT 06520, USA

6 Present Address: Centre for Biotechnology and Bioengineering, Universidad de Chile, Santiago, Chile results confirm and extend previous observations of the two holoenzymes: D1:2-PSII has a greater quantum yield of primary charge separation, faster $\left[\mathrm{P}_{680}{ }^{+} Q_{\mathrm{A}}^{-}\right]$charge recombination and less photoinhibition that results in a slower rate and higher yield of photoassembly of its apo-WOC-PSII complex. In contrast, D1:1-PSII has a lower quantum yield of primary charge separation, a slower $\left[\mathrm{P}_{680}{ }^{+} Q_{\mathrm{A}}^{-}\right]$charge recombination rate, and faster photoinhibition that together result in higher rate but lower yield of photoassembly at higher light intensities. Cyanobacterial PSII reaction centers that contain the high- and low-light D1 isoforms can tailor performance to optimize photosynthesis at varying light conditions, with similar consequences on their photoassembly kinetics and yield. These different efficiencies of photoassembly versus photoinhibition impose differential costs for biosynthesis as a function of light intensity.

Keywords Photosystem II - Oxygen evolution · Water-oxidizing complex $\cdot$ Photo-assembly ·

Photosynthetic efficiency

\section{Introduction}

The membrane-embedded Photosystem II (PSII) reaction center acts as a water-plastoquinone oxidoreductase in the light reactions of oxygenic photosynthesis (McEvoy and Brudvig 2006; Muh and Zouni 2011; Vinyard et al. 2013a). The active site for water oxidation (known as the wateroxidizing complex (WOC)) is a $\mathrm{Mn}_{4} \mathrm{Ca}_{1} \mathrm{O}_{5}$ hetero-cubane structure that is stabilized by various amino acid residues mainly from the D1 reaction center subunit (Umena et al. 2011). The WOC is assembled from free $\mathrm{Mn}^{2+}, \mathrm{Ca}^{2+}$, $\mathrm{HCO}_{3}^{-}$, water, and a terminal electron acceptor in the apoWOC-PSII complex through a process termed 


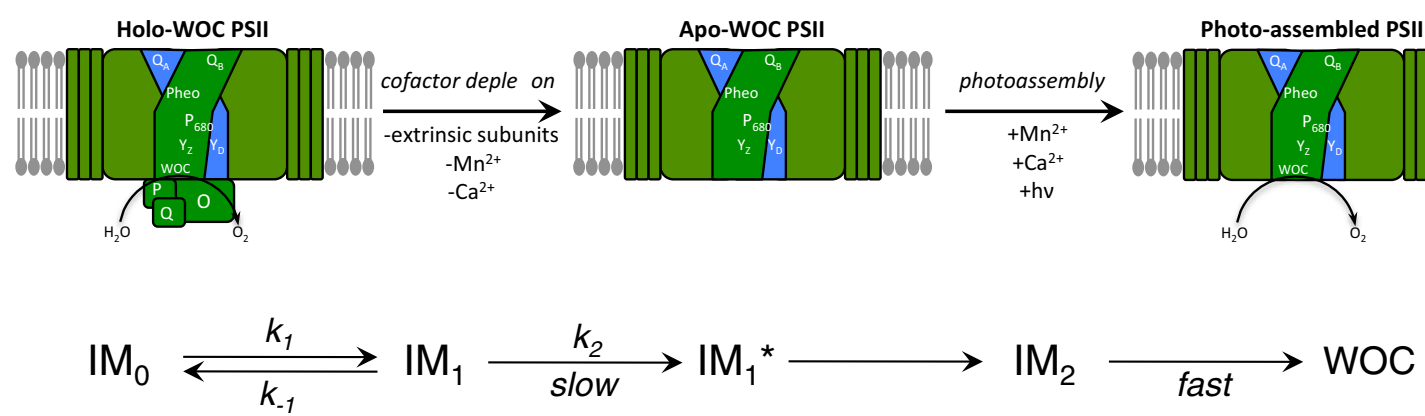

Fig. 1 Top steps involved in removal of cofactors and photoassembly of PSII. Bottom sequence of kinetic intermediates during photoassembly

photoassembly (also known as photoactivation, Fig. 1) (Tamura and Cheniae 1987; Dasgupta et al. 2008; Chen et al. 1995). Photoassembly can be experimentally probed by detecting oxygen (the terminal product of the fully assembled WOC) using short flashes of light at varying flash frequencies. This technique has enabled determination of the stoichiometry of the metal ions (Ananyev and Dismukes 1996; Zaltsman et al. 1997) and the average oxidation state of the manganese ions (number of flashes needed to produce $\mathrm{O}_{2}$ ) (Zaltsman et al. 1997; Kolling et al. 2012). In spinach and cyanobacteria, the kinetic sequence and chemical mechanism of photoassembly have been elucidated as follows (Fig. 1, reviewed in (Burnap 2004; Dasgupta et al. 2008)): Apo-WOC-PSII plus inorganic cofactors $\left(\mathrm{IM}_{0}\right)$ form the first light-induced $\mathrm{Mn}^{3+}$ intermediate $\left(\mathrm{IM}_{1}\right)$ upon oneelectron photooxidation of a $\mathrm{Mn}^{2+}$ and release of a proton; this step occurs with a quantum yield of only $0.25-0.75 \%$ in vitro (Baranov et al. 2004). Next, a slow (rate-determining) dark rearrangement step occurs in which $\mathrm{Ca}^{2+}$ binds within the ligand field of the high affinity $\mathrm{Mn}^{3+}$, forming intermediate $\mathrm{IM}_{1}$ *. $\mathrm{IM}_{2}$ is next formed following one-electron photo-oxidation of a second $\mathrm{Mn}^{2+}$, and is rapidly converted to a functional WOC when exposed to additional light and the necessary cofactors. In spinach, the rate of formation of $\mathrm{IM}_{1}$ is approximately ten-fold faster than the subsequent dark step that forms IM $_{1} *$ (Ananyev et al. 1999; Zaltsman et al. 1997). Non-functional photoassembly intermediates form reversibly with the above productive pathway, leading to lower yields of active PSII-WOC, thus indicating careful coordination of cofactor availability and photon utilization is essential in vivo.

Little is known about how variations in PSII subunit content affect WOC photoassembly. Several groups have reported significant variations in PSII-WOC activity $\left(\mathrm{O}_{2}\right.$ evolution) as a function of natural variations in the cyanobacterial reaction center D1 subunit (coded for by the psbA gene) (Vinyard et al. 2013b; Sugiura and Boussac 2014; Mulo et al. 2012). For example, in the cyanobacterium Synechococcus elongatus PCC 7942, two isoforms, D1:1 and D1:2, differ by 25 out of 360 amino acids (Golden et al. 1986) and are differentially expressed based on environmental conditions (Schaefer and Golden 1989a, b). D1:1 is expressed at low light and contains a Gln at position 130 that hydrogen bonds to the primary electron acceptor, pheophytin (Umena et al. 2011). D1:1 has more efficient WOC cycling at very low light intensities due to slower charge recombination reactions (Vinyard et al. 2013b). D1:2, which contains a Glu at position 130, is expressed at high light intensity, has faster charge recombination, and is significantly less sensitive to photoinhibition (Campbell et al. 1998; Clarke et al. 1993; Kulkarni and Golden 1994; Ogami et al. 2012; Sander et al. 2010; Tichy et al. 2003). By expressing D1:1 during normal growth conditions (low or moderate light intensities) and D1:2 during high light intensities, Synechococcus 7942 can maximize photosynthetic function in diverse light environments (Clarke et al. 1993; Vinyard et al. 2013b). In addition to D1:1 and D1:2, other D1 isoforms are present in various cyanobacteria including $\mathrm{D} 1_{m}$ (expressed under normal growth conditions in Synechocystis sp. PCC 6803) and D1' (expressed under low oxygen conditions) (reviewed in Mulo et al. 2009; Sugiura and Boussac 2014).

We previously introduced the approach of expressing D1:1 or D1:2 from Synechococcus 7942 in a transgenic psbA-deficient strain of the green alga Chlamydomonas reinhardtii (Gimpel and Mayfield 2013; Vinyard et al. 2013b). In our previous studies, the choice of a Chlamydomonas background eliminated interference from phycobiliproteins and Photosystem I-associated chlorophylls in fluorescence studies. We found that the phenotypes exhibited by D1:1- or D1:2-PSII in Synechococcus 7942 were faithfully reproduced in the green algal system (Vinyard et al. 2013b). Seven additional D1 point mutants were constructed in these backgrounds that exhibited systematic variations between these native isoforms, confirming the robustness of these variations in vivo (Vinyard et al. 2014). With these strains in hand, we hypothesized that D1 isoforms may also affect the rate and/or yield of WOC photoassembly. Using flash oximetry experiments, we show that the presence of D1:1 leads to a faster rate of photoassembled WOC, while the presence of D1:2 leads to a higher final yield of photoassembled WOC at higher 
intensities. Implications for photosynthetic efficiency are discussed.

\section{Materials and methods}

Chlamydomonas reinhardtii strains in which the native psbA copies have been interrupted and D1:1 and D1:2 isoforms from Synechococcus elongatus PCC 7942 heterologously expressed were available from a previous study (Gimpel and Mayfield 2013; Vinyard et al. 2013b, 2014). These strains were generated from wild-type CC$125\left(137 \mathrm{c}, \mathrm{mt}^{+}\right)$, which was used as a control. The three strains used in this study (D1:1, D1:2, and CC-125) have D1 proteins with identical C-terminal domains (after Ala344) to avoid differences in post-translational processing. Photoautotrophic cultures were maintained in $\mathrm{HS}$ medium (Sueoka 1960) with $5 \mathrm{mM} \mathrm{HCO}_{3}^{-}$and thylakoid membrane fragments were isolated, as previously described (Vinyard et al. 2013b). Briefly, cells were lysed using a BeadBeater (Bartlesville, OK) with zirconia beads and thylakoid membranes were isolated by centrifugation. Thylakoid membranes were resuspended in Buffer B (20 mM MES, pH 6.0, $15 \mathrm{mM} \mathrm{NaCl}, 5 \mathrm{mM} \mathrm{MgCl} 2$, and $5 \mathrm{mM}$ EDTA) and $25 \%$ Triton X-100 was slowly added to a final Triton X-100/Chl ratio of 20:1 and a Chl concentration of $2.0 \mathrm{mg} \mathrm{mL}^{-1}$. After $25 \mathrm{~min}$. of slow stirring, thylakoid membrane fragments were washed three times in Buffer B, and resuspended at $\sim 2 \mathrm{mg} \mathrm{Chl} \mathrm{mL}^{-1}$ in $25 \%$ glycerol, $300 \mathrm{mM}$ sucrose, $50 \mathrm{mM}$ MES, pH 6.0, and $35 \mathrm{mM} \mathrm{NaCl}$. Aliquots were flash frozen in liquid $\mathrm{N}_{2}$ and stored at $77 \mathrm{~K}$.

Apo-WOC-PSII was generated by first incubating $C$. reinhardtii whole cells in a reducing agent, $1 \mathrm{mM}$ hydroxylamine $\left(\mathrm{NH}_{2} \mathrm{OH}\right)$, in darkness for $1 \mathrm{~min}$ before cell lysis (Tamura and Cheniae 1987; Cheniae and Martin 1971; Hwang and Burnap 2005). After thylakoid membrane fragments were isolated, $\mathrm{NH}_{2} \mathrm{OH}$-treated samples were sequentially incubated with $200 \mathrm{mM} \mathrm{MgCl}_{2}$ in alkaline CHES buffer (20 mM, pH 9.4) for $1 \mathrm{~min}$ at room temperature to release $\mathrm{Mn}^{2+}$ and $\mathrm{Ca}^{2+}$ and the extrinsic PSII subunits. Bound PsbO slows photoassembly by hindering $\mathrm{Mn}^{2+}$ accessibility to PSII (Miyao and Murata 1984), as well as hindering complete $\mathrm{Mn}^{2+}$ release from the WOC during Mn depletion (Kuwabaraa et al. 1985). Two volumes of $50 \mathrm{mM}$ MES and $1 \mathrm{mM}$ EDTA were then added to quench the alkaline treatment and chelate released $\mathrm{Mn}^{2+}$ (modified from Hunziker et al. 1987). This optimized protocol eliminates $\mathrm{O}_{2}$ evolution below detection limit, while allowing high yields of photoassembly.

Chlorophyll was extracted in methanol and quantified using extinction coefficients from Porra (Porra et al. 1989). The concentration of PSII reaction centers was determined by electron paramagnetic resonance (EPR) detection of tyrosine-D radical $\left(\mathrm{Y}_{\mathrm{D}}^{*}\right)$. Samples were illuminated with a red LED $\left(\lambda_{\max }=623 \mathrm{~nm}, 500 \mu \mathrm{E} \mathrm{m}^{-2} \mathrm{~s}^{-1}\right)$ for $30 \mathrm{~s}$ at $0{ }^{\circ} \mathrm{C}$, dark adapted for $10 \mathrm{~min}$ at $0{ }^{\circ} \mathrm{C}$, and then quench cooled in liquid nitrogen in darkness. The double integral of the $Y_{D}$ signal was quantified against a standard curve of Fremy's salt (Babcock et al. 1983). Manganese content in each sample was also assessed via EPR. $100 \mu \mathrm{L}$ of each sample at $1.5 \mathrm{mg} \mathrm{Chl} \mathrm{mL}^{-1}$ was digested with $400 \mu \mathrm{L}$ concentrated nitric acid (trace metal analysis grade, Sigma Aldrich, St. Louis, MO) and incubated for $1 \mathrm{~h}$ at room temperature. Samples were spun down at $15,000 \times g$ and $200 \mu \mathrm{L}$ of supernatant was loaded into quartz EPR tubes. The peak-to-trough height of the first (low-field) feature of the six-line $\mathrm{Mn}^{2+}$ signal was quantified to compare relative $\mathrm{Mn}^{2+}$ content.

Flash $\mathrm{O}_{2}$ yield was measured using a homemade membrane-covered $\mathrm{O}_{2}$ electrode described previously (Ananyev and Dismukes 1996). The electrode was equipped with a pulsed LED light source $\left(\lambda_{\max }=660 \mathrm{~nm}, 85 \mu \mathrm{E} \mathrm{m}^{-2} \mathrm{~s}^{-2}\right)$ that was used to generate flashes of variable duration (5-60 ms) and flash frequency $(0.1-1 \mathrm{~Hz}, 10-1 \mathrm{~s}$ dark time between flashes).

Each flash $\mathrm{O}_{2}$ run was performed with $0.5 \mu \mathrm{M}$ PSII as thylakoid membrane fragments in a total volume of $13 \mu \mathrm{L}$. All experiments included $50 \mathrm{mM}$ MES, pH 6.0, $300 \mathrm{mM}$ sucrose, $35 \mathrm{mM} \mathrm{NaCl}$, and $2 \mathrm{mM} \mathrm{K}_{3} \mathrm{Fe}(\mathrm{CN})_{6}$. For photoassembly experiments, $10 \mathrm{mM} \mathrm{CaCl}{ }_{2}, 0.1 \mathrm{mM} \mathrm{MnCl}{ }_{2}$, and $10 \mathrm{mM} \mathrm{NaHCO}$ were added (complete photoassembly buffer).

As previously described (Baranov et al. 2004), experimental kinetics of reconstitution of $\mathrm{O}_{2}$ evolution by photoassembly exhibit lag and growth phases that were fit to a biexponential model, Eq. 1:

$Y(t)=Y_{\mathrm{O}_{2}}+A_{1} \mathrm{e}^{-\lambda_{1} t}-A_{2} \mathrm{e}^{-\lambda_{2} t}$,

where $Y(t)$ is the ensemble population of all $\mathrm{O}_{2}$ evolving centers at time $t, Y_{\mathrm{O}_{2}}$ is the maximum (steady-state) population at the end of photoassembly, and $A_{1}$ and $A_{2}$ represent the relative amplitudes of the lag $\left(\lambda_{1}\right)$ and growth $\left(\lambda_{2}\right)$ phases of $Y(t)$, respectively. This kinetic equation represents a three-step microscopic process in which a fast reversible light-induced step $\left(k_{1} / k_{-1}\right)$ is followed by a much slower (rate-limiting) dark step $\left(k_{2}\right)$, and all subsequent steps leading to an active center are fast and unresolved. At all conditions of the photoassembly experiments conducted herein, $k_{1}>>k_{2}$, and the analytical solution to Eq. (1) simplifies to $\lambda_{1}=\left(k_{1}+k_{-1}\right)$ and $\lambda_{2}=k_{2} k_{1} /$ $\left(k_{1}+k_{-1}\right)$ (Baranov et al. 2004).

Data represent the ensemble averages of three independent experiments. The averaged data were fit to Eq. 1, and error is reported as uncertainty in fits. 


\section{Results}

We elected to work with isolated thylakoid membranes instead of detergent-solubilized PSII particles to ensure results would describe native PSII centers and by avoiding possible differential detergent solubilization kinetics (unanticipated). Isolated thylakoid membrane samples (holo-WOC-PSII) exhibited steady-state $\mathrm{O}_{2}$ evolution rate at saturating light intensity of $267 \mu \mathrm{mol} \mathrm{O}_{2}(\mathrm{mg} \mathrm{Chl})^{-1} \mathrm{~h}^{-1}$ for CC-125, $161 \mu \mathrm{mol} \mathrm{O} \mathrm{O}_{2}(\mathrm{mg} \mathrm{Chl})^{-1} \mathrm{~h}^{-1}$ for D1:1-PSII, and $188 \mu \mathrm{mol} \mathrm{O} \mathrm{O}_{2}(\mathrm{mg} \mathrm{Chl})^{-1} \mathrm{~h}^{-1}$ for D1:2-PSII as measured in the standard buffer (40 mM MES, pH 6.0, $200 \mathrm{mM}$ sucrose, $10 \mathrm{mM} \mathrm{NaCl}, 10 \mathrm{mM} \mathrm{CaCl} 2,10 \mathrm{mM}$ $\mathrm{MgCl}_{2}, 0.1 \mathrm{mM} \mathrm{DCBQ}$, and $\left.2 \mathrm{mM} \mathrm{K}_{3} \mathrm{Fe}(\mathrm{CN})_{6}\right)$. The $\mathrm{Y}_{\mathrm{D}}^{-}$ radical EPR signal in holo-WOC-PSII samples was measured and quantified against a standard curve of Fremy's salt to determine absolute PSII concentrations (Fig. 2). The ratio of $\mathrm{Chl}$ to $\mathrm{Y}_{\mathrm{D}}$ (Chl/PSII) was determined to be 356 for CC-125, 487 for D1:1, and 546 for D1:2, in agreement with our previous study (Vinyard et al. 2013b).

An optimal flash duration that produces adequate $\mathrm{O}_{2}$ signal and low photoinhibition was determined for intact thylakoid membranes and found to be $30 \mathrm{~ms}$ (Fig. 3). This duration was used for all subsequent experiments. It is essentially the same duration flash as found for native spinach thylakoid membranes (Ananyev and Dismukes 1996). Shorter flashes produce insufficient time for $\mathrm{Mn}^{2+}$ binding and photooxidation, as seen by the lower yield of $\mathrm{O}_{2}$ and the greater number of flashes required to reach the steady state (see 5 vs. 15 and $30 \mathrm{~ms}$ ). Longer flash durations are less effective in restoring $\mathrm{O}_{2}$ evolution on the first flash, and all subsequent flashes inhibit further by a bimolecular process that increases with the $\mathrm{Mn}^{2+}$

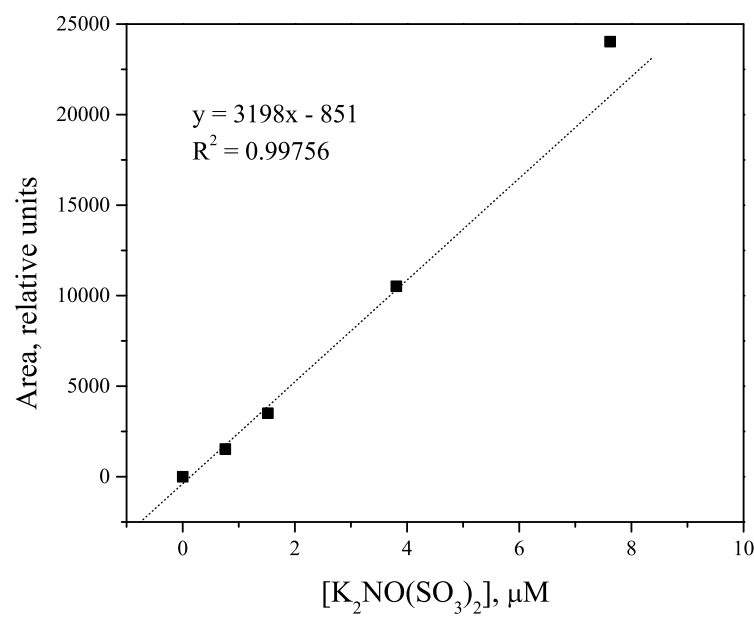

Fig. 2 Left EPR signals from Fremy's salt standards were integrated twice and used to generate a quantitative standard curve for spin concentration. Right the EPR spectra of $Y_{\mathrm{D}}$ in thylakoid membrane fragments were used to determine the PSII concentrations for concentration. This Mn-dependent inhibition by long pulses arises from photooxidation of additional $\mathrm{Mn}^{2+}$ at nonfunctional site(s) that block native assembly (Ananyev et al. 2001; Chen et al. 1995). This bimolecular inhibition process is known to be reduced by $\mathrm{Ca}^{2+}$, and on this basis has been assigned to the binding and photooxidation of $\mathrm{Mn}^{2+}$ at the $\mathrm{Ca}^{2+}$ effector site to from a misfolded protein or misassembled cluster (Ananyev et al. 2001; Chen et al. 1995).

Confirmation that a previously developed method for cofactor extraction $\left(\mathrm{NH}_{2} \mathrm{OH}\right.$ incubation followed by alkaline salt washing and final EDTA washing of thylakoid membrane fragments) also works well for $C$. reinhardtii thylakoid fragments was performed in two ways: EPR analysis of $\mathrm{Mn}^{2+}$ content, and $\mathrm{O}_{2}$ evolution of the depleted

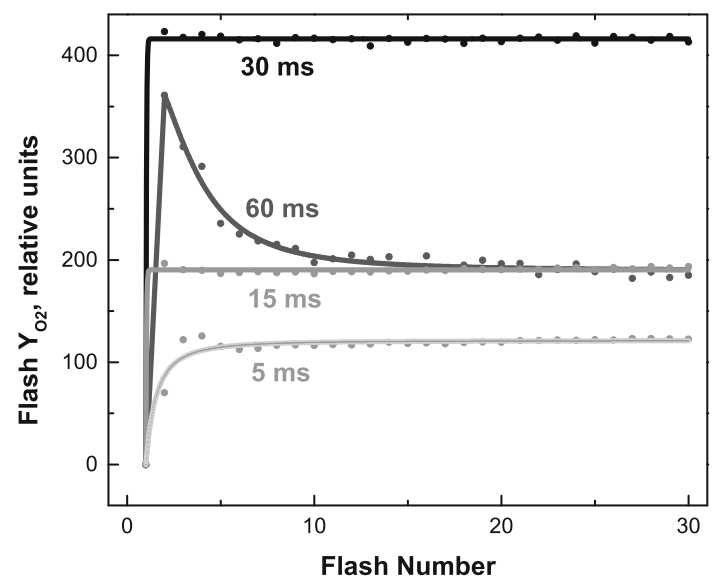

Fig. 3 Holo-WOC-PSII from CC-125 was used to optimize the LED flash duration. Flash rate from $0.33 \mathrm{~Hz} .0 .5 \mu \mathrm{M}$ PSII was suspended in $50 \mathrm{mM}$ MES, pH 6.0, $300 \mathrm{mM}$ sucrose, $35 \mathrm{mM} \mathrm{NaCl}$, and $2 \mathrm{mM}$ $\mathrm{K}_{3} \mathrm{Fe}(\mathrm{CN})_{6}$

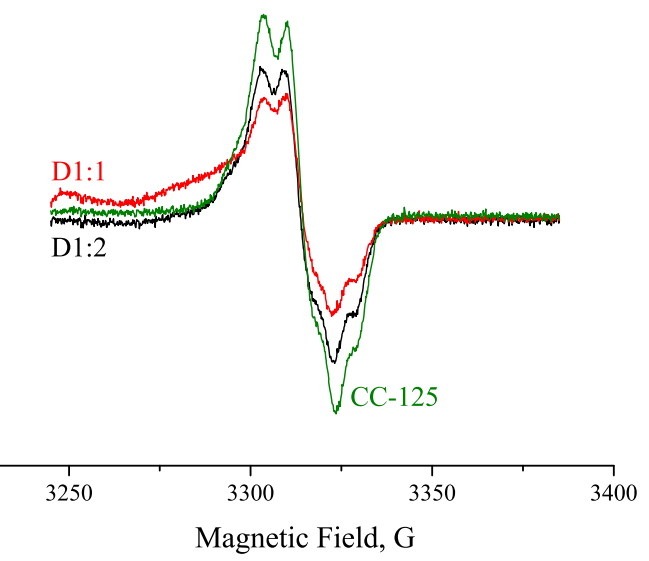

normalization of photoassembly data. All samples were analyzed at $9.45 \mathrm{GHz}$ frequency, $0.1 \mathrm{~mW}$ power, $2 \mathrm{G}$ modulation amplitude at $10 \pm 1 \mathrm{~K}$ for 32 scans 


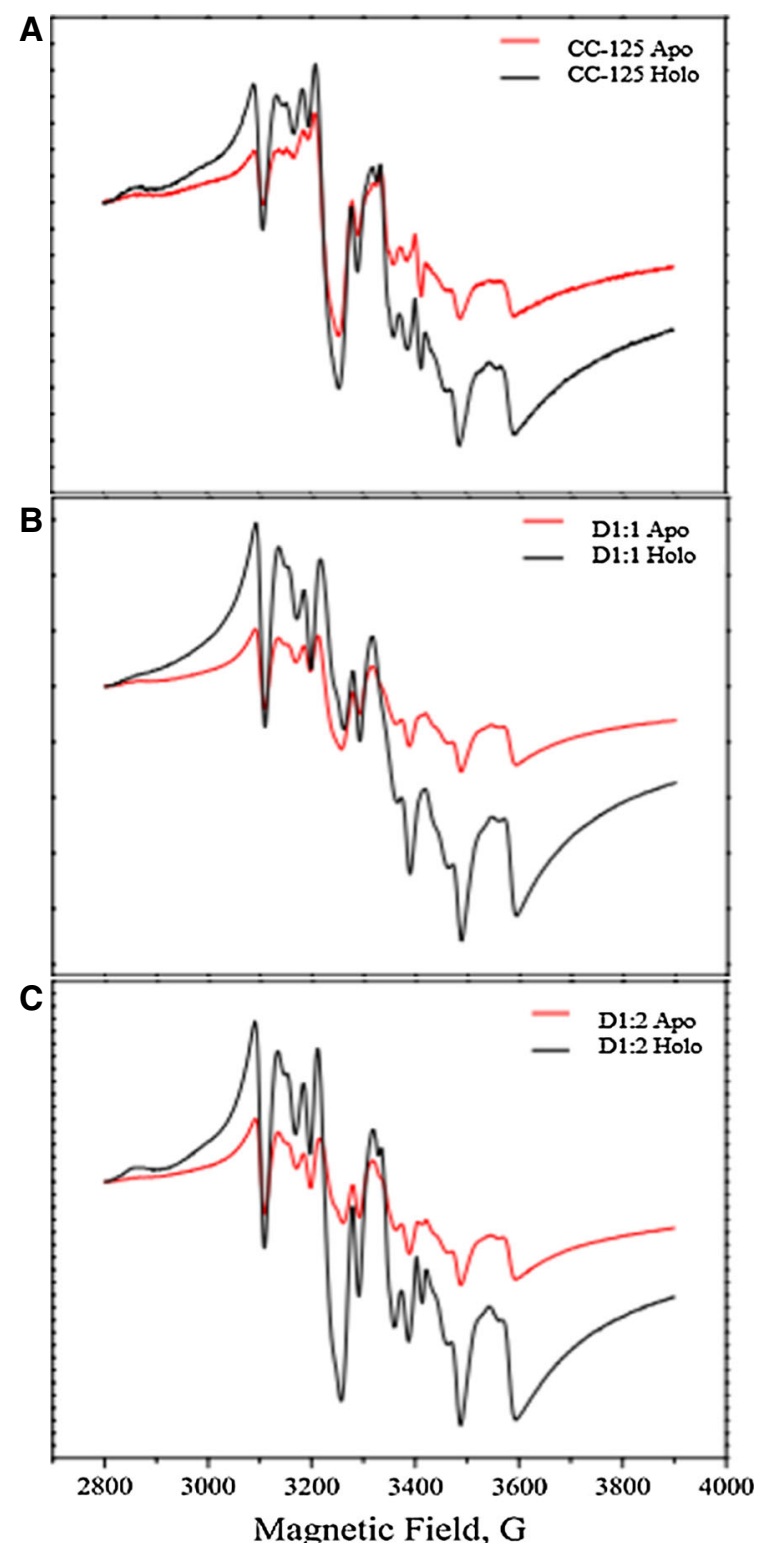

Fig. 4 a-c EPR analysis of the $\mathrm{Mn}^{2+}$ signal intensity reveals that $\sim 60 \%$ of the $\mathrm{Mn}^{2+}$ was removed from apo-WOC-PSII samples. Samples were analyzed at $9.36 \mathrm{GHz}$ frequency, $0.25 \mathrm{~mW}$ power (a and b) or $0.5 \mathrm{~mW}$ power (c), and $15 \mathrm{G}$ modulation amplitude at

thylakoid membrane fragments with incomplete cofactor addition. EPR analysis of the residual $\mathrm{Mn}^{2+}$ content of apo membrane fragments is similar: apo/holo ratios of 0.367 in CC-125, 0.392 in D1:1, and 0.420 in D1:2 (Fig. 4). The $\sim 40 \%$ residual population of $\mathrm{Mn}^{2+}$ in the apo-WOC-PSII thylakoids is consistent with earlier papers by Cheniae (1980), Blankenship and Sauer (1974), and with our prior results using spinach thylakoids membranes. The flash $\mathrm{O}_{2}$ yields of apo-WOC-PSII membranes were below the detection limit in blank buffer (50 mM MES, pH 6.0, $300 \mathrm{mM}$ sucrose, $\left.35 \mathrm{mM} \mathrm{NaCl}, 2 \mathrm{mM} \mathrm{K}_{3} \mathrm{Fe}(\mathrm{CN})_{6}\right)$ and when either $0.1 \mathrm{mM} \mathrm{Mn}{ }^{2+}$ or $10 \mathrm{mM} \mathrm{Ca}^{2+}$ was added

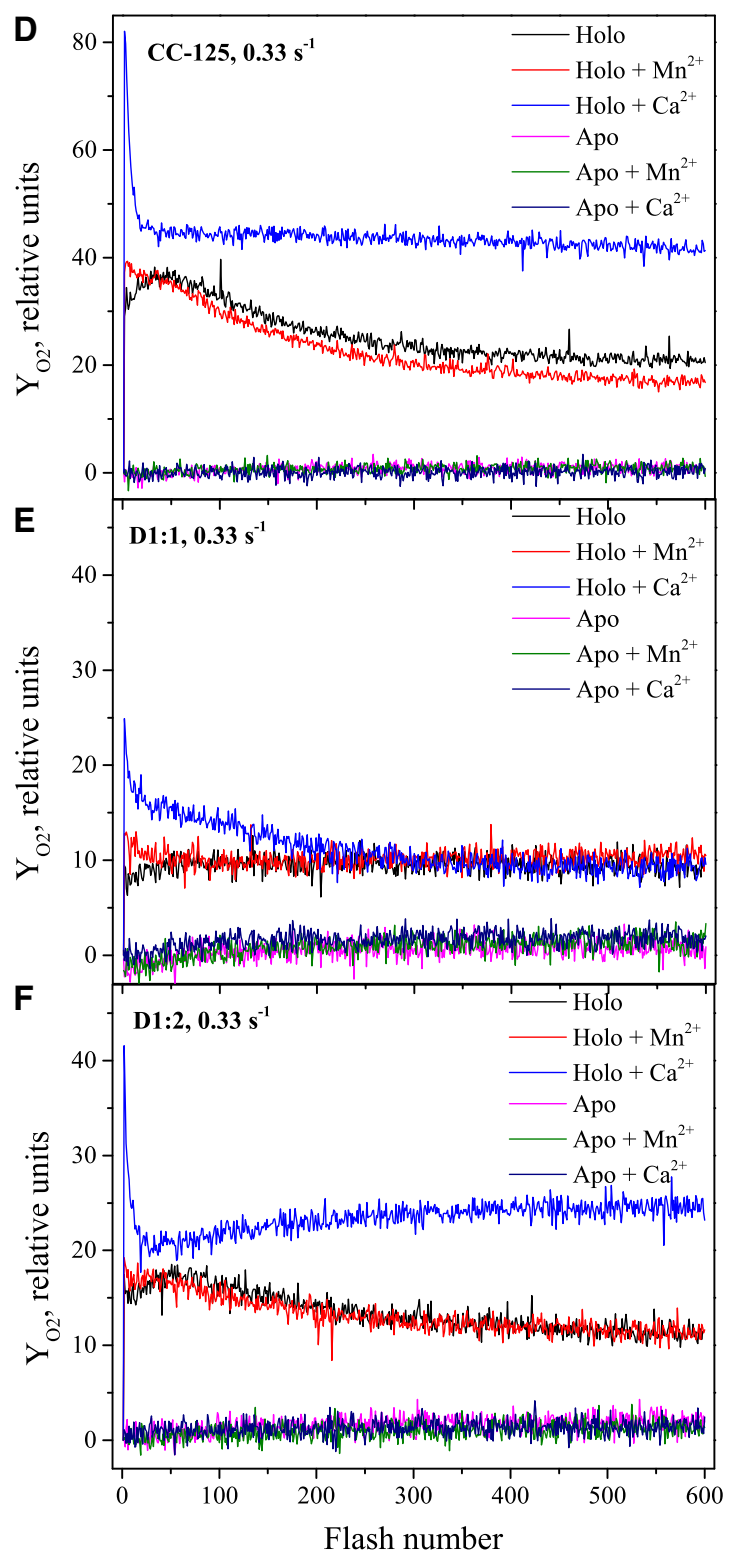

$12 \pm 1 \mathrm{~K}$. d-f Flash $\mathrm{O}_{2}$ yields $\left(Y_{\mathrm{O}_{2}}\right)$ of apo-WOC-PSII and holoWOC-PSII samples. All samples contained $0.5 \mu \mathrm{M}$ PSII in $50 \mathrm{mM}$ MES, pH 6.0, $300 \mathrm{mM}$ sucrose, $35 \mathrm{mM} \mathrm{NaCl}$, and $2 \mathrm{mM} \mathrm{K}_{3} \mathrm{Fe}(\mathrm{CN})_{6}$. $10 \mathrm{mM} \mathrm{CaCl}{ }_{2}$ or $0.1 \mathrm{mM} \mathrm{MnCl} 2$ was added as indicated

(Fig. 4). These controls demonstrate that the WOC is not functional in apo-samples, and that the residual Mn content in thylakoids is not available to reconstitute $\mathrm{O}_{2}$ evolution capacity, neither with added $\mathrm{Mn}^{2+}$ nor added $\mathrm{Ca}^{2+}$ alone.

The kinetics of photoassembly exhibit an observable lag phase prior to a mono-exponential growth phase to maximum yield and, and in some cases, light-dependent decay of the maximum yield occurs (negative slope following maximum) (Fig. 5). The latter decay is due to photoinhibition (Ananyev et al. 2001; Baranov et al. 2004). The photoinhibition contribution was typically small or zero during the photoassembly period and could be removed by 
linear extrapolation of the negative slope following the peak in photoassembly to zero time (dashed line in Fig. 5). This correction for photoinhibition was always larger in CC-125-PSII and D1:1-PSII than in D1:2-PSII samples.
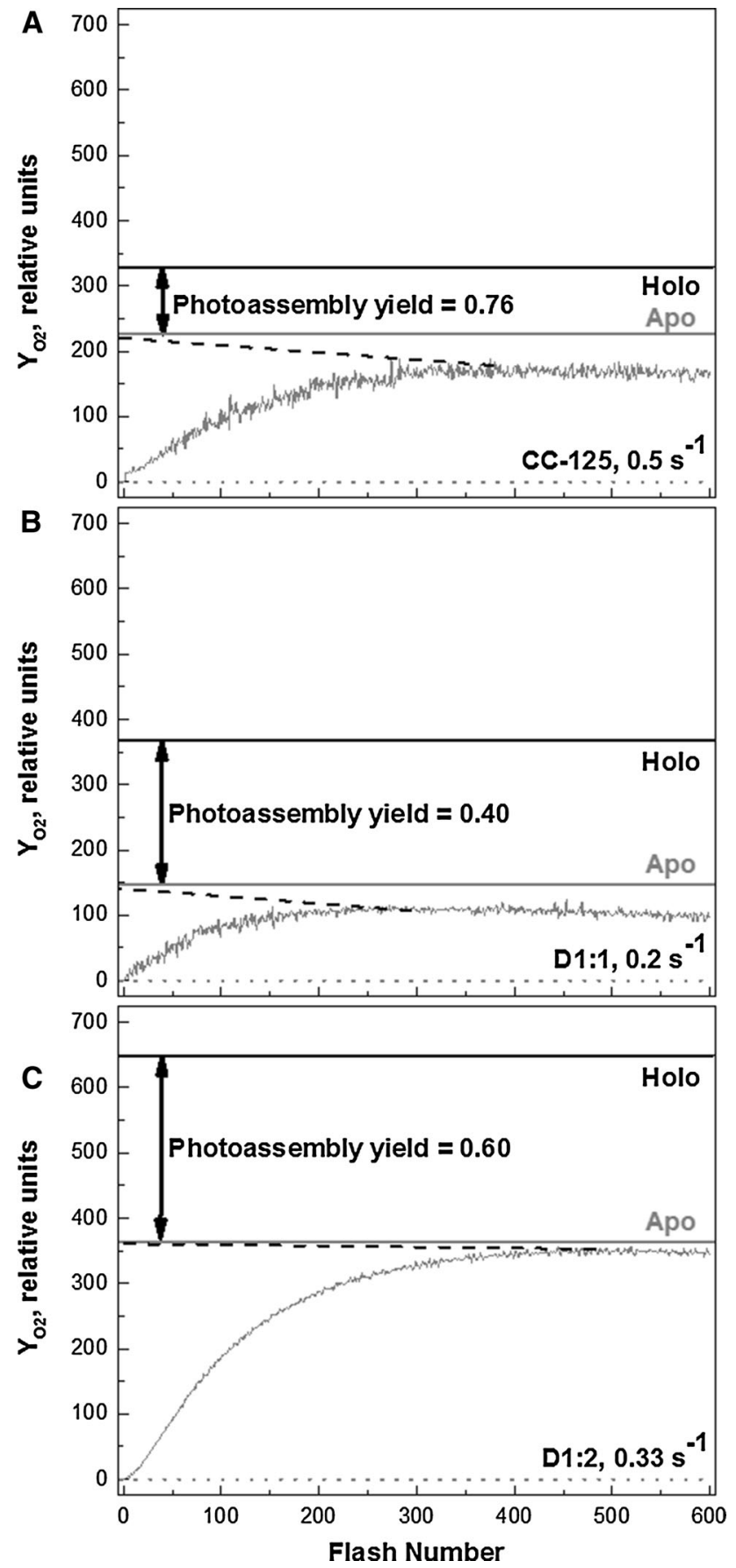

Fig. 5 Apo-WOC-PSII samples for CC-125 (a), D1:1 (b), and D1:2 (c) were compared at the flash frequencies which gives the highest observed photoassembly yields ( 0.2 and $0.33 \mathrm{~s}^{-1}$, respectively). $0.5 \mu \mathrm{M}$ of holo-WOC-PSII (black lines) or apo-WOC-PSII (gray traces) was suspended in complete photoassembly buffer. PSII was quantified using $Y_{\mathrm{D}}$ content (Fig. 2). The maximal flash oxygen yield was corrected for photoinhibition by linear extrapolation of the post maximum decay (dashed lines)
The flash frequency was varied from $0.1 \mathrm{~Hz}$ to $1 \mathrm{~Hz}$ in steps to simulate the transition from low to high light intensities, and the steady-state $\mathrm{O}_{2}$ yields were determined. As illustrated in Fig. 5, after approximately 400 flashes, a steady-state yield is reached and CC-125-PSII and D1:2PSII produce significantly more $\mathrm{O}_{2}$ than D1:1-PSII at the chosen flash rate. Comparison of the flash rate dependence of $Y_{\mathrm{O}_{2}}$, given in Fig. 6a, shows that D1:2-PSII photoassembles a higher yield of centers when using faster

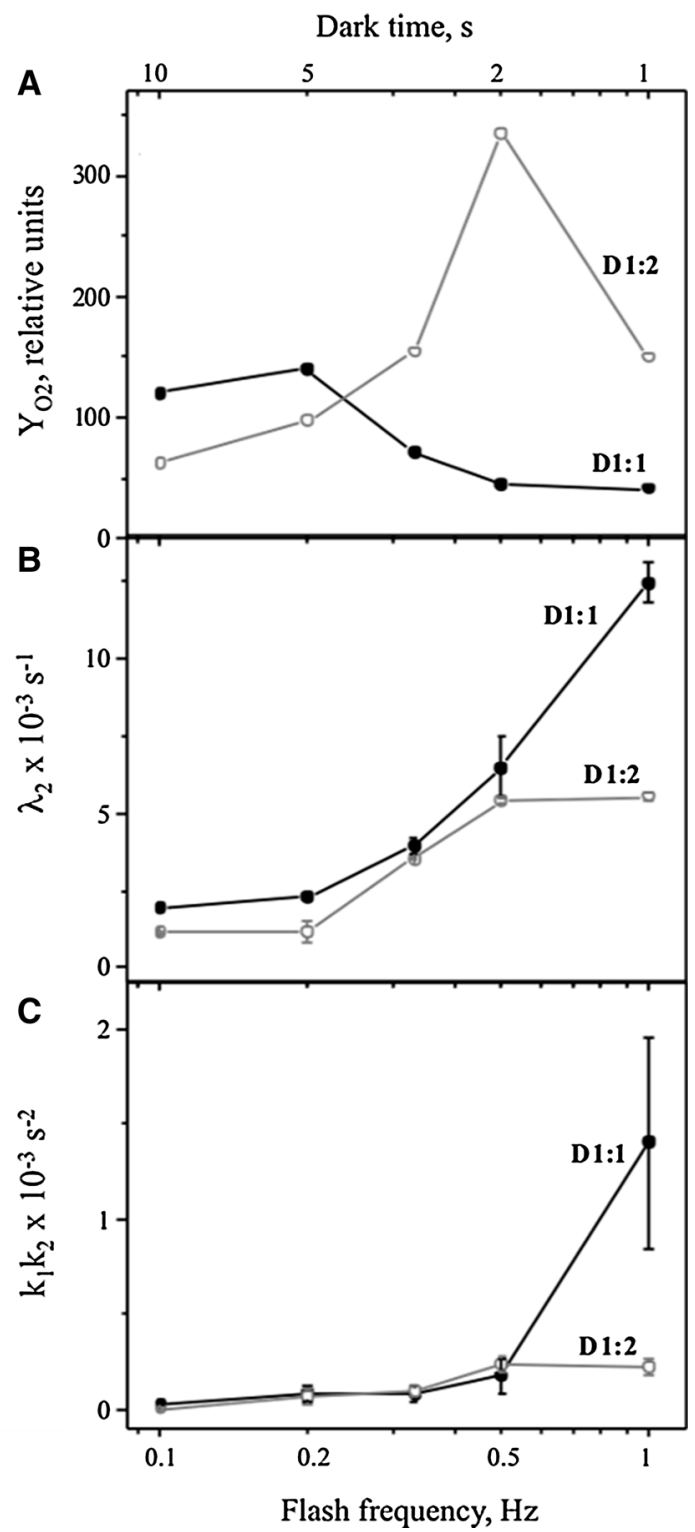

Fig. 6 D1:1 apo-WOC-PSII (closed symbols) or D1:2 apo-WOCPSII (open symbols) was photoassembled using $30 \mathrm{~ms}$ flashes applied at $0.1-1 \mathrm{~Hz}$ (resulting in $10-1 \mathrm{~s}$ dark time between flashes) in complete photoassembly buffer. a steady-state photoassembly yield, b rate constant $\lambda_{2}$ representing the rate-limiting assembly step, and c $k_{1} k_{2}\left(=\lambda_{1} \lambda_{2}\right)$ representing the product of forward rate constants from $\mathrm{IM}_{0}$ to $\mathrm{IM}_{1}$ * 
flash frequencies ( $\geq 0.33 \mathrm{~Hz}$ ), while D1:1-PSII exhibits its maximal $\mathrm{O}_{2}$ yield at slower flash frequencies, from $0.2 \mathrm{~Hz}$ and below. The maximum ratio of photoassembled $\mathrm{O}_{2}$ yield $\left(Y_{\mathrm{O}_{2}}\right)$ between D1:2 and D1:1 is $7: 1$ at $0.5 \mathrm{~Hz}$ and $1: 2$ at $0.1 \mathrm{~Hz}$ flash rate, respectively (Fig. 6a), indicating a strong dependence on the average light intensity. For comparison, at high light intensity, the D1:2-PSII holoenzyme has been previously shown to have a $16-40 \%$ higher steady-state turnover frequency for $\mathrm{O}_{2}$ production than D1:1-PSII holoenzyme in vitro (Sugiura et al. 2008; Vinyard et al. 2013b) and a 50-70\% higher primary charge separation efficiency (equal to the primary photochemical quantum yield, $\Phi$ ) in vivo (Vinyard et al. 2014). These differences in relative photochemical rate for the holoenzymes versus the photoassembly reaction follow the same pattern but quantitatively differ, suggesting other contributions. This should be expected considering the complexity of the photoassembly process which is known to produce misassembled centers (depending upon the cofactor concentrations) that do not contribute to $\mathrm{O}_{2}$ recovery initially, but may be reversibly reactivated (Ananyev et al. 2001; Chen et al. 1995; Dasgupta et al. 2008)

Figure 5 compares the steady-state flash $\mathrm{O}_{2}$ yield of photoassembled apo-WOC-PSII centers to that for the intact holo-WOC-PSII, prior to cofactor extraction, at the indicated flash rates. This comparison shows that the final $\mathrm{O}_{2}$ flash yields of the photoassembled centers are approximately $76 \%$ for CC-125, $60 \%$ for D1:2, and $40 \%$ for D1:1. These yields are quite high considering that the absence of the extrinsic PsbO subunit alone lowers the flash $\mathrm{O}_{2}$ yield by approximately $30 \%$ in spinach (Hunziker et al. 1987; Tamura and Cheniae 1987; Zaltsman et al. 1997). All three extrinsic subunits of PSII are missing from apo-WOC-PSII samples.

The kinetics of photoassembly exhibit both lag and growth phases in agreement with previous results from spinach thylakoids and grana membranes (Ananyev et al. 2001; Baranov et al. 2004). As before, the time-dependent yield, $Y(t)$, was fitted to the biexponential expression shown as Eq. 1. This model provides excellent numerical fits with low residuals ( $R^{2}$ in the range $0.923-0.998$; Table 1$)$. The "assembly phase" macroscopic rate constant, $\lambda_{2}$, obtained from these fits is rate-limiting for photoassembly and is plotted as a function of flash rate in Fig. 6b. For both D1:1PSII and D1:2-PSII, the fastest photoassembly rate occurs at $1 \mathrm{~Hz}$ flash rate (the fastest rate examined) and decreases with decreasing flash frequencies. This trend is as expected for a light intensity-dependent process of low quantum yield, and is consistent with earlier behavior seen for spinach (Baranov et al. 2004; Zaltsman et al. 1997). D1:1 assembles at comparable or slightly faster rate than D1:2 at all flash rates examined below $0.5 \mathrm{~Hz}$ (Fig. 6b). As the flash rate increases to $1 \mathrm{~Hz}, \mathrm{D} 1: 1$ photoassembles at 2.3fold faster rate compared to D1:2.

The value of $\lambda_{1}$, the "lag phase" macroscopic rate constant, is approximately 7-9-fold faster than $\lambda_{2}$, while its amplitude $\left(A_{1}\right)$ contributes only a small fraction $(11.5 \%$ average) of the total signal ( $A_{1}$ is 8 - to 13 -fold smaller than $A_{2}$ over the range of flash frequencies studied; Table 1). This outcome is by choice as we deliberately used high concentrations of $\mathrm{Mn}^{2+}$ and $\mathrm{Ca}^{2+}$ relative to the apoWOC-PSII concentration to ensure high photoassembly yields and low photoinhibition rates (Baranov et al. 2004). High $\mathrm{Mn}^{2+}$ and $\mathrm{Ca}^{2+}$ concentrations directly contribute to shortening the lag phase in spinach thylakoids and grana membranes, making it difficult to measure with precision (Baranov et al. 2004). Under these conditions, the precision of measuring $\lambda_{1}$ is insufficient to allow a statistically significant difference between $\mathrm{D} 1: 1$ and $\mathrm{D} 1: 2$ to be determined.

\section{Discussion}

The data show that the two natural D1 isoforms offer clear advantages at different light intensities for assembling active PSII-WOC centers capable of supporting photosynthesis through water oxidation. This process provides the primary energy equivalents for cellular growth in the form of reducing equivalents (electrons) and proton gradient. D1:1-PSII assembles more WOC centers at low light flux, while D1:2-PSII assembles more WOC centers at high light flux. D1:1 assembles at rates equal to or faster than D1:2 at all measured light intensities, while becoming significantly faster at higher light intensities $(1 \mathrm{~Hz})$ where the rate of photooxidation to form $\mathrm{Mn}^{3+}$ in $\mathrm{IM}_{1}$ (given by microscopic rate constant $k_{1}$, Fig. 1) dominates over the dark charge recombination reaction (microscopic rate constant $\left.k_{-1}\right)$. However, at these high light intensities, D1:1 suffers from faster photoinhibition than does D1:2, significantly limiting is overall yield of activated centers.

The kinetic parameters $\lambda_{1}$ and $\lambda_{2}$ in Eq. 1 can be used to obtain the microscopic rate constants $k_{1}$ and $k_{2}$ in the reaction model (Fig. 1) as described above. Here we see that the two isoforms are kinetically indistinguishable in terms of their forward product fluxes at flash rates of $0.5 \mathrm{~Hz}$ and slower. From these expressions, we obtain the product of the forward rate constants, $k_{1} k_{2}=\lambda_{1} \lambda_{2}$, which is plotted as a function of flash rate in Fig. 6c. This product may also be thought of as the inverse of the product of transit times required to go from $\mathrm{IM}_{0}$ to $\mathrm{IM}_{1} *$ without any recombination. Hence, the only explanation for the greater final yield of photoassembled D1:1-PSII-WOC centers at these slower flash rates is because of a slower charge recombination rate $\left(k_{-1}\right)$. This interpretation of the 
Table 1 Kinetic fitting parameters of photoassembly at varying flash frequencies

\begin{tabular}{lcrrrrrrrr}
\hline & \multicolumn{1}{l}{$y_{0}$} & \multicolumn{1}{c}{$A_{1}$} & $\begin{array}{c}A_{1} \\
(\%)\end{array}$ & $\begin{array}{l}\lambda_{1} \times 10^{-3} \\
\left(\mathrm{~s}^{-1}\right)\end{array}$ & \multicolumn{1}{c}{$A_{2}$} & $\begin{array}{l}A_{2} \\
\%)\end{array}$ & $\begin{array}{l}\lambda_{2} \times 10^{-3} \\
\left(\mathrm{~s}^{-1}\right)\end{array}$ & $\begin{array}{c}R^{2} \\
\lambda_{1} \times \lambda_{2} \times 10^{-3} \\
\left(\mathrm{~s}^{-2}\right)\end{array}$ \\
\hline $\mathrm{D} 1: 1$ & & & & & & & & & \\
$1 \mathrm{~Hz}$ & $41.3 \pm 0.4$ & $5.9 \pm 2.2$ & 11.7 & $113 \pm 44.1$ & $-44.9 \pm 1.6$ & 88.3 & $12.4 \pm 0.650$ & 0.9480 & $1.40 \pm 0.553$ \\
$0.5 \mathrm{~Hz}$ & $43.8 \pm 1.6$ & $14.0 \pm 7.0$ & 19.9 & $27.4 \pm 12.5$ & $-56.3 \pm 6.1$ & 80.1 & $6.48 \pm 1.00$ & 0.9780 & $0.177 \pm 0.0854$ \\
$0.33 \mathrm{~Hz}$ & $71.3 \pm 0.9$ & $11.9 \pm 4.0$ & 12.4 & $21.5 \pm 10.1$ & $-84.6 \pm 3.7$ & 87.6 & $3.97 \pm 0.252$ & 0.9844 & $0.0854 \pm 0.0405$ \\
$0.2 \mathrm{~Hz}$ & $140.0 \pm 0.5$ & $11.6 \pm 3.7$ & 7.3 & $37.2 \pm 20.3$ & $-148.4 \pm 1.3$ & 92.7 & $2.29 \pm 0.0391$ & 0.9913 & $0.0852 \pm 0.0466$ \\
$0.1 \mathrm{~Hz}$ & $120.2 \pm 0.4$ & $17.3 \pm 1.9$ & 11.3 & $14.4 \pm 3.11$ & $-136.0 \pm 1.6$ & 88.7 & $1.97 \pm 0.0344$ & 0.9977 & $0.0283 \pm 0.00615$ \\
$\mathrm{D} 1: 2$ & & & & & & & & & \\
$1 \mathrm{~Hz}$ & $149.9 \pm 0.5$ & $19.1 \pm 1.9$ & 10.1 & $40.3 \pm 7.35$ & $-169.0 \pm 1.6$ & 89.9 & $5.54 \pm 0.0912$ & 0.9956 & $0.223 \pm 0.0409$ \\
$0.5 \mathrm{~Hz}$ & $334.8 \pm 1.2$ & $55.2 \pm 4.8$ & 12.4 & $43.9 \pm 7.6$ & $-390.1 \pm 3.8$ & 87.9 & $5.40 \pm 0.0921$ & 0.9966 & $0.237 \pm 0.0412$ \\
$0.33 \mathrm{~Hz}$ & $154.0 \pm 0.3$ & $20.6 \pm 3.0$ & 10.5 & $27.6 \pm 7.91$ & $-176.2 \pm 2.4$ & 89.5 & $3.54 \pm 0.0541$ & 0.9901 & $0.0975 \pm 0.0280$ \\
$0.2 \mathrm{~Hz}$ & $97.4 \pm 18.5$ & $7.5 \pm 7.7$ & 6.7 & $61.3 \pm 37.4$ & $-104.2 \pm 16.7$ & 93.3 & $1.17 \pm 0.325$ & 0.9225 & $0.0717 \pm 0.0480$ \\
$0.1 \mathrm{~Hz}$ & $62.0 \pm 0.2$ & $10.4 \pm 2.0$ & 12.6 & $5.98 \pm 1.81$ & $-72.2 \pm 2.06$ & 87.4 & $1.15 \pm 0.0341$ & 0.9900 & $0.00688 \pm 0.00209$ \\
\hline
\end{tabular}

Data from three replicates were ensemble averaged and then fit to the function $Y_{\mathrm{O}_{2}}=y_{0}+A_{1} \mathrm{e}^{-\lambda_{1} x}-A_{2} \mathrm{e}^{-\lambda_{2} x}$

photoassembly kinetics fits precisely with the experimentally measured slower charge recombination rate of the D1:1-PSII holoenzyme which is dominated by $\left[Q_{\mathrm{A}}^{-} \mathrm{P}_{680}{ }^{+}\right]$ recombination (Vinyard et al. 2013b; Sugiura and Boussac 2014; Vinyard et al. 2014). Figure 6c also shows that above $0.5 \mathrm{~Hz}$ flash rate, D1:1 has a faster forward flux from $\mathrm{IM}_{0}$ to $\mathrm{IM}_{1} *$ than does $\mathrm{D} 1: 2$ (seven-fold larger at $1 \mathrm{~Hz}$ flash rate), but produces fewer active centers than does D1:2 (Fig. 6a). The only way this can happen is if the high light flux causes greater photoinhibition in D1:1. Again, this agrees with the experimentally determined faster photoinhibition rate of D1:1-PSII holoenzyme (Vinyard et al. 2013b; Sugiura and Boussac 2014; Vinyard et al. 2014).

Previous studies in isolated PSII reaction centers from Thermosynechococcus elongatus BP-1 containing either D1:1 or D1:2 have revealed that the midpoint potentials of both Pheo/Pheo ${ }^{-}$(Kato et al. 2009; Sugiura et al. 2010) and $Q_{\mathrm{A}} / Q_{\mathrm{A}}^{-}$(Kato et al. 2012; Ogami et al. 2012) are significantly more negative in D1:1-PSII. These differences have been used to explain the higher quantum yield of primary charge separation in D1:2-PSII (larger free energy gap between $\mathrm{P}_{680}^{*} / \mathrm{P}_{680}{ }^{+}$and $\left.\mathrm{Pheo} / \mathrm{Pheo}^{-}\right)$and the slower recombination events in D1:1-PSII (dominated by $\left[Q_{\mathrm{A}}^{-} \mathrm{P}_{680}{ }^{+}\right]$recombination) (Vinyard et al. 2013b; Sugiura and Boussac 2014; Vinyard et al. 2014). These traits are likely responsible for the functional advantage in photoassembly kinetics for cells containing D1:1 in low-light conditions, and cells containing D1:2 in high light conditions (Vinyard et al. 2013b).

While PSII centers containing D1:2 have higher photoassembly yields at high light intensities (Fig. 6a), the rate of photoassembly is significantly slower than centers containing D1:1 (Fig. 6b, c). We note that maximal photosynthetic efficiency would be achieved with both a high yield and a fast rate. However, in order to maintain efficient photo-protection in the holoenzyme, the D1:2 isoform pays the price of slower WOC photoassembly.

In this study, we have extended the knowledge of the relative advantages of D1:1 versus D1:2 to include photoassembly yields and kinetics. The higher photoassembly yield observed for D1:2-PSII correlates with its higher quantum yield of charge separation which provides greater protection against photoinhibition. By contrast, in D1:1PSII, WOC assembly is faster at low light intensities due to slower $\left[Q_{\mathrm{A}}^{-} \mathrm{P}_{680}{ }^{+}\right]$recombination kinetics. We postulate that a faster rate of photoassembly may provide an evolutionary advantage for cyanobacteria expressing the D1:1 isoform in low-light environments. It is significant to note that cyanobacteria, that can express these two isoforms of D1 in response to environmental cues, must pay appreciably different energy costs to assemble the same number of functional PSII centers at high light intensity where the substantially faster photoinhibition rate of D1:1 greatly limits the yield of functional PSII centers. These data affirm that Nature has developed a mechanism for maintaining water oxidation capability under varying light regimens and thus another mechanism for controlling the efficiency of photosynthesis.

Acknowledgments This work was supported by grants from the National Science Foundation-Chemistry of Life Processes, CHE1213772 (to GCD), and the U.S. Department of Energy, Consortium for Algal Biofuels Commercialization, Grant DE-EE0003373 (to SPM). JSS was supported by the Waksman Institute of Microbiology and the Aresty Research Center for Undergraduates at Rutgers University. DJV was supported by the Department of Deference Army Research Office through a National Defense Science and Engineering Graduate (NDSEG - 32CFR168a), and a National Science Foundation Graduate Research Fellowship, DGE-0937373. JG was supported by the Comisión Nacional de Investigación Científica y Tecnológica de Chile (CONICYT). We thank Clyde Cady for assistance with EPR measurements. 


\section{References}

Ananyev GM, Dismukes GC (1996) Assembly of the tetra-Mn site of photosynthetic water oxidation by photoactivation: Mn stoichiometry and detection of a new intermediate. Biochemistry 35(13):4102-4109. doi:10.1021/bi952667h

Ananyev GM, Murphy A, Abe Y, Dismukes GC (1999) Remarkable affinity and selectivity for $\mathrm{Cs}^{+}$and uranyl $\left(\mathrm{UO}_{2}{ }^{2+}\right)$ binding to the manganese site of the apo-water oxidation complex of Photosystem II. Biochemistry 38(22):7200-7209. doi:10.1021/ bi990023u

Ananyev GM, Zaltsman L, Vasko C, Dismukes GC (2001) The inorganic biochemistry of photosynthetic oxygen evolution/ water oxidation. Biochim Biophys Acta 1503(1-2):52-68

Babcock GT, Ghanotakis DF, Ke B, Diner BA (1983) Electron donation to Photosystem II in reaction center preparations. Biochim Biophys Acta 723(2):276-286

Baranov SV, Tyryshkin AM, Katz D, Dismukes GC, Ananyev G, Klimov VV (2004) Bicarbonate is a native cofactor for assembly of the manganese cluster of the photosynthetic water oxidizing complex. Kinetics of reconstitution of oxygen evolution by photoactivation. Biochemistry 43(7):2070-2079

Blankenship RE, Sauer K (1974) Manganese in photosynthetic oxygen evolution. I. Electron paramagnetic resonance study of the environment of manganese in Tris-washed chloroplasts. Biochim Biophys Acta 357(2):252-266

Burnap RL (2004) D1 protein processing and Mn cluster assembly in light of the emerging Photosystem II structure. Phys Chem Chem Phys 6(20):4803-4809

Campbell D, Eriksson M-J, Oquist G, Gustafsson P, Clarke AK (1998) The cyanobacterium Synechococcus resists UV-B by exchanging photosystem II reaction-center D1 proteins. Proc Natl Acad Sci USA 95(1):364-369

Chen C, Kazimir Janet, Cheniae GM (1995) Calcium modulates the photoassembly of Photosystem I1 (Mn)4-clusters by preventing ligation of nonfunctional high-valency states of manganese. Biochemistry 34(41):13511-13526

Cheniae GM (1980) Manganese binding sites and presumed manganese proteins in chloroplasts, vol 69., Methods in enzymology Academic Press, New York

Cheniae GM, Martin IF (1971) Effects of hydroxylamine on Photosystem II: I. Factors affecting the decay of $\mathrm{O}_{2}$ evolution. Plant Physiol 47(4):568-575

Clarke AK, Soitamo A, Gustafsson P, Oquist G (1993) Rapid interchange between two distinct forms of cyanobacterial photosystem II reaction-center protein D1 in response to photoinhibition. Proc Natl Acad Sci USA 90(21):9973-9977

Dasgupta J, Ananyev GM, Dismukes GC (2008) Photoassembly of the water-oxidizing complex in photosystem II. Coord Chem Rev 252(3-4):347-360. doi:10.1016/j.ccr.2007.08.022

Gimpel J, Mayfield S (2013) Analysis of heterologous regulatory and coding regions in algal chloroplasts. Appl Microbiol Biotechnol 97(10):4499-4510. doi:10.1007/s00253-012-4580-4

Golden SS, Brusslan J, Haselkorn R (1986) Expression of a family of $p s b A$ genes encoding a photosystem II polypeptide in the cyanobacterium Anacystis nidulans R2. EMBO J 5(11):2789-2798

Hunziker D, Abramowicz DR, Damoder R, Dismukes GC (1987) Evidence for an association between a $33 \mathrm{kDa}$ extrinsic membrane protein, manganese and photosynthetic oxygen evolution. I. Correlation with the $\mathrm{S}_{2}$ multiline EPR signal. Biochim Biophys Acta 890(1):6-14

Hwang HJ, Burnap RL (2005) Multiflash experiments reveal a new kinetic phase of photosystem II manganese cluster assembly in Synechocystis sp PCC 6803 in vivo. Biochemistry 44(28): 9766-9774
Kato Y, Shibamoto T, Yamamoto S, Watanabe T, Ishida N, Sugiura M, Rappaport F (1817) Boussac A (2012) Influence of the PsbA1/PsbA3, $\mathrm{Ca}^{2+} / \mathrm{Sr}^{2+}$ and $\mathrm{Cl}^{-} / \mathrm{Br}^{-}$exchanges on the redox potential of the primary quinone $\mathrm{Q}_{\mathrm{A}}$ in Photosystem II from Thermosynechococcus elongatus as revealed by spectroelectrochemistry. Biochim Biophys Acta 11:1998-2004

Kato Y, Sugiura M, Oda A, Watanabe T (2009) Spectroelectrochemical determination of the redox potential of pheophytin a, the primary electron acceptor in photosystem II. Proc Natl Acad Sci USA 106(41):17365-17370. doi:10.1073/pnas.0905388106

Kolling DRJ, Cox N, Ananyev GM, Pace RJ, Dismukes GC (2012) What are the oxidation states of manganese required to catalyze photosynthetic water oxidation? Biophys J 103(2):313-322. doi:10.1016/j.bpj.2012.05.031

Kulkarni RD, Golden SS (1994) Adaptation to high light intensity in Synechococcus sp. strain PCC 7942: regulation of three psbA genes and two forms of the D1 protein. J Bacteriol 176(4): 959-965

Kuwabaraa T, Miyaob Mitsue, Murataa Teruyo, Murata Norio (1985) The function of 33-kDa protein in the photosynthetic oxygenevolution system studied by reconstitution experiments. Biochim Biophys Acta-Bioenerg 806(2):283-289

McEvoy JP, Brudvig GW (2006) Water-splitting chemistry of photosystem II. Chem Rev 106(11):4455-4483. doi:10.1021/ cr0204294

Miyao M, Murata Norio (1984) Role of the 33-kDa polypeptide in preserving $\mathrm{Mn}$ in the photosynthetic oxygen-evolution system and its replacement by chloride ions. FEBS Lett 170(2):350-354

Muh F, Zouni A (2011) Light-induced water oxidation in Photosystem II. Front Biosci 16:3072-3132

Mulo P, Sicora C, Aro E-M (2009) Cyanobacterial $p s b A$ gene family: optimization of oxygenic photosynthesis. Cell Mol Life Sci 66(23):3697-3710. doi:10.1007/s00018-009-0103-6

Mulo P, Sakurai I, Aro E (2012) Strategies for $p s b A$ gene expression in cyanobacteria, green algae and higher plants: from transcription to PSII repair. Biochim Biophys Acta 1817(1):247

Ogami S, Boussac A, Sugiura M (2012) Deactivation processes in PsbA1-Photosystem II and PsbA3-Photosystem II under photoinhibitory conditions in the cyanobacterium Thermosynechococcus elongatus. Biochim Biophys Acta 1817(8):1322-1330

Porra RJ, Thompson WA, Kriedemann PE (1989) Determination of accurate extinction coefficients and simultaneous equations for assaying chlorophylls $a$ and $b$ extracted with four different solvents: verification of the concentration of chlorophyll standards by atomic absorption spectroscopy. Biochim Biophys Acta 975(3):384-394

Sander J, Nowaczyk M, Buchta J, Dau H, Vass I, Deak Z, Dorogi M, Iwai M, Rogner M (2010) Functional characterization and quantification of the alternative $p s b A$ copies in Thermosynechococcus elongatus and their role in photoprotection. J Biol Chem 285(39):29851-29856. doi:10.1074/jbc.M110.127142

Schaefer MR, Golden SS (1989a) Differential expression of members of a cyanobacterial $p s b A$ gene family in response to light. J Bacteriol 171(7):3973-3981

Schaefer MR, Golden SS (1989b) Light availability influences the ratio of two forms of D1 in cyanobacterial thylakoids. J Biol Chem 264(13):7412-7417

Sueoka N (1960) Mitotic replication of deoxyribonucleic acid in Chlamydomonas reinhardi. Proc Natl Acad Sci USA 46(1):83-91

Sugiura M, Boussac A (2014) Some Photosystem II properties depending on the D1 protein variants in Thermosynechococcus elongatus. Biochim Biophys Acta 1837:1427-1434

Sugiura M, Boussac A, Noguchi T, Rappaport F (2008) Influence of Histidine-198 of the D1 subunit on the properties of the primary electron donor, P680, of photosystem II in Thermosynechococcus 
elongatus. Biochim Biophys Acta 1777(4):331-342. doi:10.1016/ j.bbabio.2008.01.007

Sugiura M, Iwai E, Hayashi H, Boussac A (2010) Differences in the interactions between the subunits of Photosystem II dependent on D1 protein variants in the thermophilic cyanobacterium Thermosynechococcus elongatus. J Biol Chem 285(39): 30008-30018. doi:10.1074/jbc.M110.136945

Tamura N, Cheniae GM (1987) Photoactivation of the wateroxidizing complex in Photosystem II membranes depleted of $\mathrm{Mn}$ and extrinsic proteins. I. Biochemical and kinetic characterization. Biochim Biophys Acta 890:179-194

Tichy M, Lupinkova L, Sicora C, Vass I, Kuvikova S, Prasil O, Komenda J (2003) Synechocystis 6803 mutants expressing distinct forms of the Photosystem II D1 protein from Synechococcus 7942: relationship between the psbA coding region and sensitivity to visible and UV-B radiation. Biochim Biophys Acta 1605(1-3):55-66

Umena Y, Kawakami K, Shen J-R, Kamiya N (2011) Crystal structure of oxygen-evolving Photosystem II at a resolution of 1.9 angstrom. Nature 473(7345):55-60
Vinyard DJ, Ananyev GM, Dismukes GC (2013a) Photosystem II: the reaction center of oxygenic photosynthesis. Annu Rev Biochem 82(1):577-606. doi:10.1146/annurev-biochem-070511-100425

Vinyard DJ, Gimpel J, Ananyev GM, Cornejo MA, Golden SS, Mayfield SP, Dismukes GC (2013b) Natural variants of Photosystem II subunit D1 tune photochemical fitness to solar intensity. J Biol Chem 288(8):5451-5462. doi:10.1074/jbc. M112.394668

Vinyard DJ, Gimpel J, Ananyev GM, Mayfield SP, Dismukes GC (2014) Engineered Photosystem II reaction centers optimize photochemistry versus photoprotection at different solar intensities. J Am Chem Soc 136(10):4048-4055. doi:10.1021/ja50 02967

Zaltsman L, Ananyev G, Bruntrager E, Dismukes GC (1997) Quantitative kinetic model for photoassembly of the photosynthetic water oxidase from its inorganic constituents: requirements for manganese and calcium in the kinetically resolved steps. Biochemistry 36:8914-8922 